1 INTRODUCTION

\title{
Absolute Spacetime: The Twentieth Century Ether
}

\author{
Carl H. Brans"
}

\section{Introduction}

Gauge theories, to which Friedrich Hehl has contributed so much, explore the mysterious fundamental role which symmetries play in our understanding of the physical world. To have a symmetry we need two parts: something fixed while something else changes. Much of the progress of modern physical theories has come as a result of studying this "fixed/changing" dichotomy, analyzing it and suggesting new paradigms. For most of the history

\footnotetext{
${ }^{1}$ Loyola University, New Orleans, LA 70118, email:brans@loyno.edu
}

\begin{abstract}
All gauge theories need "something fixed" even as "something changes." Underlying the implementation of these ideas all major physical theories "something fixed," i.e., absolute. This model must provide at least the fol. the review an ap"hole" argument. This scenario, which does not seem to be widely known to practicing relativists, is nevertheless still interesting in terms of its impact for fundamental gauge issues.
\end{abstract}


of physics, space, and more recently spacetime, has in some sense or other been the underlying fixed object on which theories are written and in terms of which experimental results are reported. The active, changing, part of the symmetry, the gauge group, consists of coordinate changes, or to use more contemporary terminology, diffeomorphisms. Yet, in spite of its uncontrovertible central role, a thorough understanding of spacetime models is still one of the most elusive goals of modern physics.

In this paper, I would like to review questions related to these issues using the now discredited ether models of the eighteenth and nineteenth centuries for comparison. In the earlier times, the ether was some notdirectly-observable-substratum thought to be needed by certain theories. For example, in the Newtonian gravitational precursor to field theory, Newton thought that action at a distance was "... so great an absurdity, that...no man, who has in philosophical matters a competent faculty for thinking, can ever fall into it." [1]. He speculated that in the case of gravitation, the force may be produced by varying densities of the mechanical ether in the presence of gravitating masses. In later periods it played a more passive role, providing a fixed, preferred reference system relative to which velocities should be measured for calculations of Lorentz forces and current sources in Maxwell equations. We will not be concerned with the actual details of these old ether models here, but only use them as a backdrop to consider contemporary questions revolving around observability issues in physical models. The interested reader is invited to consult the massive two volume work on the ether by Whittaker [2].

Those of us who work day by day in theoretical physics and especially relativity may tend to take for granted the huge package of assumptions that we impose on our spacetime models, most of which cannot be supported by direct experiment. It is this tacit acceptance of unobservable properties of our model that motivates this paper. Of course, these assumptions have been questioned by many workers from the time of Greek physics to the present. Max Jammer has given us an excellent review of this subject [3]. Without any claim to completeness we can also note more contemporary work, for example, Connes [4], Rovelli [5], Madore and Saeger [6], Heller and Sasin [7], Brans [8]. Other participants of this meeting have contributed to this field, including Rosenbaum [9] in his lecture to this meeting, and Lämmerzahl and Macias 10].

Many of these questions border on the philosophical, and philosophers and historians of science have certainly made their contributions. Again the literature is too huge to survey here. I mention only the work of Grünbaum [11] and Earman [12. 


\section{Contemporary Spacetime Models}

In almost all theories a model of spacetime, say $M$, is required with at least the following properties:

- Point set. That is, $M$ contains atomic elements, $p \in M$, representing idealized point events. The existence and identity of this structure is absolutely necessary for all of the following ones.

- Topological manifold. $M$ must have a topology such that it is locally Euclidean. That is, each $p$ must lie in a neighborhood homeomorphic to $\mathbb{R}^{n}$ for some integer $n$. Again, this property is needed for the others.

- Smooth manifold. $M$, in addition to being locally homeomorphic to $\mathbb{R}^{n}$, must be locally diffeomorphic to it. The local diffeomorphisms constitute the local coordinates needed to express smooth functions and to operate on them differentially.

- Geometric manifold. $M$ must carry a smooth metric, connection and perhaps, as Hehl has taught us, torsion.

- Bundle structures. Finally, additional gauge structures and fields require local patching together of products of $M$ with models of the field/gauge group space.

The advent of supersymmetry, etc. surely makes this list incomplete, but it does provide some idea of the extensive and detailed structure that spacetime models use. Here we want to point out that this involved logical construction is built from bricks that are as essentially unobservable as were the vortices and atoms of the mechanistic ether of earlier times. It is in this sense that the title of this talk compares spacetime to the ether. But before getting into the details of these foundational lacunae, let us look at the basic gauge group of spacetime relativity, starting from its historical roots.

\section{The Classical Ether}

Trautman 13] has suggested a helpful way to look at the progress of relativity in spacetime physics using modern terminology. For more philosophical and historical details along this path see Jammer [3]. Starting with Aristotelian physics we can consider the basic spacetime model, $M$, as a simple direct product of space with time, each having some intrinsic, absolute properties,

$$
M=\text { space } \times \text { time }=\mathbb{R}^{3} \times \mathbb{R}^{1} .
$$

In this context, what passed for dynamics was defined by ascribing of natural places to specific types of matter, "earthy" things to the earth, etc. 
Skipping centuries of interesting intellectual history, we arrive at the relativity of Galilei and Newton. In actuality, as Jammer points out, Newton was strongly attached to the idea of some absolute nature for space, something more along the lines of (1). Nevertheless the formalism of his mechanics logically leads to a replacement of (1) with something like

$$
M=\text { Bundle }= \begin{cases}\text { space }=\mathbb{R}^{3} \longrightarrow & M \\ & \downarrow p \\ & \mathbb{R}^{1}=\text { time }\end{cases}
$$

Thus, spacetime, $M$ is a bundle over time, $\mathbb{R}^{1}$, with space as fiber, $\mathbb{R}^{3}$. What distinguishes bundles from ordinary products as in (1) is the absence of any natural identification of the fiber over one base point with that over some other base point. Each fiber, "space at a given time," is isomorphic to $\mathbb{R}^{3}$, but in no natural or canonical way. In classical mechanics, the fiber group, relativity gauge group $G$, is Galilean group. This is essentially the real linear affine group of dimension three. We can complete this picture to describe classical mechanics in modern terms by adding a preferred flat Euclidean metric on the fiber, three-space, and a preferred linear structure on the base space, time. Finally, we need a corresponding bundle connection whose geodesics provide the paths of free particles.

Thus, the formalism of Newtonian mechanics is more naturally associated with a bundle structure as in (2), rather than with absolute space structure, (1). Nevertheless, Newton felt strongly drawn to the latter, perhaps in part because of his difficulties with action-at-a-distance, as illustrated in the quotation in the Introduction. Thus, to Newton, space needed some mechanical properties to enable it to transfer force and energy over distances in field theories. This mechanical structure must be associated with some "substance," the ether, which incidentally provides an absolute rest frame, or the reduction of the bundle (2) to the trivial product, (1).

The attractiveness of such an absolute space model was reinforced with the advent of the unified field theory of electromagnetism. In this theory velocity appears twice. First in the Lorentz force law,

$$
\mathbf{F}=q(\mathbf{E}+\mathbf{v} \times \mathbf{B})
$$

where $\mathbf{v}$ is the velocity of the charge $q$ being acted on by the electromagnetic field, $(\mathbf{E}, \mathbf{B})$. The question left hanging is: "velocity relative to what reference frame?" Secondly, the velocity $\mathbf{v}$ appears in the source field equation

$$
\nabla \times \mathbf{B}=\mu_{0} \rho \mathbf{v}+\frac{1}{c^{2}} \frac{\partial \mathbf{E}}{\partial t},
$$

along with the new quantity of dimension speed, $c \equiv 1 / \sqrt{\epsilon_{0} \mu_{0}}$. Again, the question of what reference frame should be used to measure the source current velocity arises. Furthermore the new, unexpected speed, $c$, reappears as 


\section{END OF THE CLASSICAL ETHER: SPECIAL RELATIVITY5}

the speed of electromagnetic waves in one of the consequences of the vacuum field equations,

$$
\left(\nabla^{2}-\frac{1}{c^{2}} \frac{\partial^{2}}{\partial t^{2}}\right)\left\{\begin{array}{l}
\mathbf{E} \\
\mathbf{B}
\end{array}\right\}=0 .
$$

So, Maxwell's unified field theory of electromagnetism leaves us with three speeds, that of the source of the fields, (4), that of the object on which the fields act, (3), and the field waves themselves, (5). The Galilean relativity in (2) must then break down, since the presence of such speeds breaks its invariance, and we return to some absolute space model, (1), where space is now the "luminiferous" ether, with spacetime

$$
M=\text { ether } \times \text { time } .
$$

Of course, there were strong voices in opposition to the notion of absolute space, most notable Bishop Berkeley [14 and later Mach who referred to the notion of absolute space as a "conceptual monstrosity" 115. Einstein claimed that such ideas were instrumental in the evolution of his thinking about relativity.

At this point it might be appropriate to recall all of the effort that was put into the design of mechanical or pseudo-mechanical models of such an ether [2. It is natural to wonder how all of the work of contemporary physics involving elaborate spacetime structures and superstructures may likewise appear in the next century.

\section{End of the Classical Ether: Special Relativity}

But of course some hundred years ago Michelson and Morley results forced serious rethinking of the classical ether-space model, (6). While Lorentz and others attempted to preserve the ether by proposing length contractions and clock dilations as a result of motion through it, Einstein cut to the heart of the matter in his principle of special relativity, closely tied to the principle of operationalism which informally claims that if something conspires successfully against its observation, then its existence should not be used as part of a physical theory. Thus, if the ether's only claim to existence is as an absolute rest reference frame, and its properties make motion through it unobservable, then from the viewpoint of physics it doesn't exist.

In fact, Einstein taught us to think in terms of a unified spacetime model, with no preferred a priori splitting (apart from the qualitative space-like, time-like, light like ones) of space from time. The transformation group preserving these spacetime properties, the gauge group of special relativity is of course the Poincaré group, that is, the homogenous Lorentz group plus translations. Friedrich Hehl and his colleagues have been leaders in emphasizing the importance of this group especially in the context of general relativity and its generalizations, 16, 17], 18]. 


\section{GENERAL COVARIANCE: EINSTEIN'S RELATIVITY 6}

With the many successes of special relativity, it seems that the ether has finally been put to rest. Indeed it has in this classical sense. If you can't observe it, it doesn't exist is a standard motto. Or to paraphrase an old axiom: " No stuff has existence until it is observed to have existence." But, should we not apply this to "stuff" =manifold properties? So, is spacetime the new ether? Clearly, it does not play the same mechanical role of "transmitter of forces," as the vortex constituted stuff of the old mechanical one. Also, it clearly does not provide an "absolute rest reference frame." But, it does have other, similar properties. It provides, in operationally unobservable ways, the substratum to carry the many structures used by modern theories. and it is the point of this paper that spacetime structures in modern theories comprise a replacement for it and so have become a "new ether."

\section{General Covariance: Einstein's Relativity}

In addition to the Principle of Equivalence, which we will not consider, the Principle of General Relativity and Mach's Principle, are generally taken as foundations in models of how Einstein arrived at General Relativity. Of course, the actual history is more complicated and interesting, and the reader can consult the volume one of the Einstein Studies, [19], for a deep and accurate account of the story.

For our purposes, it is sufficient to point out that Einstein was aware of the rigid structure still remaining on the spacetime of special relativity by the Lorentz metric and the associated preferred set of inertial reference frames. Mach's Principle addresses the issue of why the fixed stars have constant velocity in the inertial frames, while the Principle of General Relativity proposes extending the physically acceptable frames beyond this restricted set. In other words, while special relativity had weakened the assumption of a preferred (zero) absolute-velocity-defining ether, it replaced it by a preferred (zero) absolute-acceleration-defining one. So, in the spirit of this paper, the next step toward generally covariant theories was a result of re-examining and loosening previous rigid structures. John Norton [20 has given us an thorough and highly interesting analysis of how Einstein arrived at his equations of General Relativity. Here we will only skim over the issue of the identity of spacetime points as illustrated in Einstein's hole dilemma. (See also [5]).

Consider a model universe, with matter and metric fields, $T, g$ on a manifold containing a region $U$, which will be the "hole." Einstein was thinking in coordinates, so let $T(x), g(x)$ be expression of solution field equations in terms of coordinates (global) $x$.

Now re-coordinate, $x \rightarrow x^{\prime}$, with $x=x^{\prime}$ in $U$, but not everywhere. Then $T^{\prime}\left(x^{\prime}\right), g^{\prime}\left(x^{\prime}\right)$ is also a solution, with

$$
g^{\prime}\left(x^{\prime}\right)=g^{\prime}(x)=g(x),
$$




\section{GENERAL COVARIANCE: EINSTEIN'S RELATIVITY 7}

within $U$. But matter and fields are different outside of $U$. So, Einstein was deflected from seeking a generally covariant theory since the following fact would seem to be paradoxical: matter outside of $U$ does not determine the fields inside of $U$ uniquely (or vice versa). At this point we must be cautious about treating this as trivial, since we are so accustomed to accepting general covariance as an obvious desideratum. From the viewpoint of development of the theory, there is more here than confusion about coordination.

In fact, it does seem on first glance that Einstein and Grossman were confused about the expression of the same metric merely displayed in different coordinates. For example

$$
d s^{2}=d x^{2}+d y^{2},
$$

or

$$
d s^{\prime 2}=\cosh ^{2}\left(x^{\prime}\right) d x^{\prime 2}+d y^{\prime 2} .
$$

Clearly (8) and (9) represent the same metric, and Einstein was aware of this. However changing the notation in (9) results in

$$
d s^{\prime 2}=\cosh ^{2}(x) d x^{2}+d y^{2} .
$$

If we then identify the points of the manifold with the pair $(x, y)$, then (8) and (10) are truly different metrics in some sense, although they are diffeomorphic (isometric). In fact it is possible to define point= "ordered pair of numbers," not "diffeomorphism equivalence class of ordered pair of numbers in each coordinate system."

This discussion highlights the difference between the active and passive interpretations of the transformation (diffeomorphism). Actually, as discussed in detail by Norton [20], Einstein's fourth presentation of this argument shows that rather than being confused at the difference between (8) and (9), he was laying the groundwork for the modern interpretation of diffeomorphisms as physical gauge transformations.

What may be surprising about this is that it seems to rob the individual points of their identity, in the absence of a metric. In other words, if $P_{1}, P_{2}$ are two points in a manifold, some diffeomorphism maps one into the other. The geometry and fields around $P_{1}$ become those around $P_{2}$, in physically equivalent geometric manifolds, so $P_{1}$ cannot be distinguished from $P_{2}$. Rovelli, [5] discusses this subject in some detail, distinguishing spacetime models as $M_{L}$, "local," with a particular smoothness and "atlas" as opposed to $M_{N}$, "non-local," which is equivalence class of all $M_{L}$ under diffeomorphisms (gauge transformations).2 However, let us recall that this discussion concerns the mathematical model which is mapped by some assumed "diffeomorphism" onto an absolute point set, spacetime. In fact, without some underlying pointset, there can be no notion of diffeomorphism.

\footnotetext{
${ }^{2}$ Corresponding to this, mathematicians distinguish "smooth structure" from "smooth manifold." We will discuss this later.
} 


\section{ABSOLUTE SPACETIME: QUANTUM THEORY 8}

Nevertheless, the idea remains that the use of diffeomorphism as physically unobservable gauge "wipes out" the individual identity of points. In fact, in discussing his final generally covariant field equations Einstein said in a letter to Schlick in 1915, "thereby time and space lose the last remnant of physical reality. All that remains is that the world is to be conceived as a four-dimensional (hyperbolic) continuum of 4 dimensions." [21]. Our point here is that this continuum carries at least as much structure as the replaced ether.

\section{Absolute Spacetime: Quantum Theory}

There still remains the list of absolute spacetime properties described in the introduction such as topology, smoothness, etc., which seem to be arbitrarily chosen. This leads to the question of regarding the role of spacetime as object or scratch pad. This is a question certainly bordering on philosophy philosophy, but also closely related to the operational foundations of quantum theory.

The principal distinguishing characteristic of quantum logic as opposed to classical logic is that in quantum theory questions correspond to projection operators in Hilbert space. The logical operators, "or," $\vee$, corresponds to "span of vector space union," while "and," $\wedge$, corresponds to intersection. This results in a non-Boolean algebra,

$$
a \wedge(b \vee c) \neq(a \wedge b) \vee(a \wedge c),
$$

By contrast classical questions concern "set-inclusion," so "or," $\vee$, becomes set union, $\cup$, and "and," $\wedge$, becomes $\cap$, set intersection. Thus, for point sets,

$$
a \cap(b \cup c)=(a \cap b) \cup(a \cap c) .
$$

In other words, quantum logic is not in general consistent with the logic of set-inclusion, which is fundamental to point-set questions.

For further discussion of these questions, see Brans [8], and especially Marlow 22]. Maybe there is not enough in this bare-bones quantum logic approach to work with (produce a theory), but, many others, too many to mention here, have also looked into the influence of quantum theory on spacetime point set properties in different ways. However the contributions

of Connes [4] and Madore [23] stand out as leading to much current work in this area.

\section{Absolute Spacetime: Choice of Smoothness}

Until recently this was thought to be trivially determined by the topology, at least for relatively simple manifolds such as $\mathbb{R}^{4}$. However, it is not trivial. In fact, some of the most exciting developments of differential topology recently 
have come as a result of what can be termed "exotic smoothness" on spaces of relatively trivial topology, for example $\mathbb{R}^{4}$. In many respects, the development of this subject parallels that of non-Euclidean and then differential geometry. Thus, for many years there were conjectures about the uniqueness of Euclidean geometry, both mathematically and as physics. Similarly, but more recently, there have been conjectures that there is essentially only one way to do calculus globally on topologically simple manifolds. The phrase "how to do calculus globally" corresponds to what mathematicians call a differentiable or smoothness structure. Physically such a structure is a global system of reference frame patches covering all of spacetime smoothly, that is, with smooth $\left(C^{\infty}\right)$ coordinate transformations in their overlaps. The phrase, "essentially only one" means only one equivalence class under diffeomorphisms of the manifold. This difference between different smoothness structures and non diffeomorphic ones can be a slippery concept to master, but is central to an understanding of differential topology. In a way, it is parallel to that involved in Einstein's hole argument discussed above. Just as the choice of different coordinates may make the metric look different when the underlying geometry is actually the same, so will a recoordination make the differential structure appear to be different, when in fact it is equivalent (diffeomorphic). This equivalence inducing class of diffeomorphisms corresponds to the underlying principle of general relativity.

By direct calculation, it is possible to show that, up to diffeomorphisms, there is only one smoothness structure on each $\mathbb{R}^{n}$ for $n=1,2,3$. For $n>4$ the same result was obtained later making use of cobordism techniques. However, the case $n=4$ remained an open one. Because of the topological triviality it was natural to conjecture that it too would turn out to be trivial with respect to differential topology. Thus, it was a tremendous surprise when as a result of the work of Donaldson, Freedman and others it was established that

Theorem; (Donaldson, Freedman, et al.) There are an infinity of smooth manifolds of topology $\mathbb{R}^{4}$, none of which are in the diffeomorphism class of the any other (including the standard one).

Thus, the diffeomorphism gauge does not cover the entire range of physics on topologically trivial $\mathbb{R}^{4}$ ! Do these provide new structures for new physics? See Brans 24] for a general review of these topics, and Asselmeyer [25 for a specific suggestion of physical content.

\section{Conclusions}

In this paper we tried to survey some of the extensive structures used on contemporary spacetime models, noting their direct physical unobservability and reflect on this rigid fine structure in the light of the historical parallel of the luminiferous ether. A hundred or so years ago, it was generally thought (apart perhaps from a few people like Gauss) that geometry was 
"pre-physics," a natural given. Now, we have take it for granted that geometric structures carry physical fields.

Perhaps it is now appropriate to speculate that the mathematical structures "point set" (e.g., Boolean, or non-Boolean...), "topological" (e.g., nonEuclidean...), "smoothness" (e.g., exotic...), etc., might also serve to carry physical properties in a manner analogous to that of "geometry."

\section{Acknowledgments}

I am indebted to John Norton and Carlo Rovelli for very helpful comments, suggestions and insights. Also, this work was partially supported by a grant, LaSpace, R150253.

Finally, of course, we are all grateful to Friedrich Hehl for his persistent clarification of the role of various spacetime gauge structures.

\section{References}

[1] I. Newton, quote taken from page 68, volume 1 of [2]

[2] E. Whittaker(1951):A History of the Theories of aether and Electricity Thomas Nelson and Sons, London, second edition.

[3] M. Jammer(1993):Concepts of Space, third edition, Dover, (1993)

[4] A. Connes(1994): Noncommutative Geometry, English edition, Academic Press (1994)

[5] C. Rovelli (1991): what is observable in classical and quantum gravity?, Class. quantum Grav. 8, 297(1991).

[6] J. Madore, L. A. Saeger(1997): Topology at the Planck Length grqc9708053.

[7] M. Heller, W. Sasin (1997): Rigorous Model of Classical Spacetime Foam, Int. J. Theo. Phys. 36, 1441 (1997)

[8] C. Brans (1980): Roles of Space-time Models, page 27 in Quantum Theory and Gravitation, ed. A. R. Marlow, Academic Press (1980).

[9] M. Rosenbaum (1998): Contribution to this volume.

[10] C. Lämmerzahl, A. Macias (1993): On the Dimensionality of SpaceTime, J. Math. Phys. 34, 4540; Addendum: J. Math. Phys. 36, 2131 (1993).

[11] A. Grünbaum (1977): in Foundations of Space Time Theories, ed J. Earman, University of Minnesota Press, 1977. 
[12] J. Earman (1990): World Enough and Space-Time : Absolute Vs. Relational Theories of Space and Time, MIT Press, 1990.

[13] A. Trautman (1970): Fibre Bundles Associated with Spacetime, Rep. Math. Phys. 1, 29(1970).

[14] See page 108 of Jammer [3].

[15] See page 143 of Jammer [3].

[16] F. Hehl, P. Von der Heyde, G. Kerlick, J. Nester(1976):General relativity with spin and torsion: foundations and prospects, Rev. Mod. Phys. 48, 393 91976).

[17] E. Mielke (1987): Geometrodynamics of Gauge Fields, AkademieVerlag(1987)

[18] F. Hehl, J. McCrea, E. Mielke, Y Ne'eman (1995): Metric-Affine gauge theory of gravity: field equations, Noether identities, world spinors, and breaking of dilation invariance, Physics Reports, 258, 1(1995)

[19] D. Howard, J. Stachel (editors) (1989): Einstein and the History of General Relativity, Birkhäuser (1989)

[20] J. Norton (1989): How Einstein found his field equations, page 101 in [19.

[21] A. Einstein (1915): Quote found on page 85 of [19].

[22] A. Marlow (1980): Empirical topology: topologies from partially ordered sets, Int. J. Theo. Phys. 19, 515(1980)

[23] J. Madore (1995):An Introduction to Noncommutative Differential Geometry and its Physical Applications, Cambridge (1995).

[24] C. Brans(1994): J. Math. Phys. 35, 5494, (1994).

[25] T. Asselmeyer (1997): Generation of source terms in general relativity by differential structures, Class. Quantum Grav. 14, 749 (1997). 